\title{
Electroencephalographic effects of ketamine on power, cross-frequency coupling, and connectivity in the alpha bandwidth
}

\author{
Stefanie Blain-Moraes ${ }^{1}$, UnCheol Lee ${ }^{1}$, SeungWoo $\mathrm{Ku}^{2}$, GyuJeong Noh ${ }^{2}$ and George A. Mashour ${ }^{1,3 *}$ \\ 1 Department of Anesthesiology, Center for Consciousness Science, University of Michigan Medical School, Ann Arbor, MI, USA \\ 2 Department of Anesthesiology, Asan Medical Center, University of Ulsan College of Medicine, Seoul, South Korea \\ ${ }^{3}$ Neuroscience Graduate Program, University of Michigan Medical School, Ann Arbor, MI, USA
}

\author{
Edited by: \\ Anthony G. Hudetz, Medical College \\ of Wisconsin, USA \\ Reviewed by: \\ Bruce Maclver, Stanford University, \\ USA \\ Jamie Sleigh, University of \\ Auckland, New Zealand \\ *Correspondence: \\ George A. Mashour, Department of \\ Anesthesiology, Center for \\ Consciousness Science, University \\ of Michigan Medical School, $1 \mathrm{H} 247$ \\ UH/SPC-5048, 1500 East Medical \\ Center Drive, Ann Arbor, MI \\ 48109-5048, USA \\ e-mail: gmashour@med.umich.edu
}

\begin{abstract}
Recent studies of propofol-induced unconsciousness have identified characteristic properties of electroencephalographic alpha rhythms that may be mediated by drug activity at $\gamma$-aminobutyric acid (GABA) receptors in the thalamus. However, the effect of ketamine (a primarily non-GABAergic anesthetic drug) on alpha oscillations has not been systematically evaluated. We analyzed the electroencephalogram of 28 surgical patients during consciousness and ketamine-induced unconsciousness with a focus on frontal power, frontal cross-frequency coupling, frontal-parietal functional connectivity (measured by coherence and phase lag index), and frontal-to-parietal directional connectivity (measured by directed phase lag index) in the alpha bandwidth. Unlike past studies of propofol, ketamine-induced unconsciousness was not associated with increases in the power of frontal alpha rhythms, characteristic cross-frequency coupling patterns of frontal alpha power and slow-oscillation phase, or decreases in coherence in the alpha bandwidth. Like past studies of propofol using undirected and directed phase lag index, ketamine reduced frontal-parietal (functional) and frontal-to-parietal (directional) connectivity in the alpha bandwidth. These results suggest that directional connectivity changes in the alpha bandwidth may be state-related markers of unconsciousness induced by both GABAergic and non-GABAergic anesthetics.
\end{abstract}

Keywords: ketamine, consciousness, general anesthesia, anesthetic-induced unconsciousness, anesthetic mechanisms

\section{INTRODUCTION}

Ketamine is an anesthetic drug that was introduced into clinical practice in the 1960s (Corssen and Domino, 1966) and is currently used for the induction of unconsciousness and, at subanesthetic doses, the prevention of acute pain or the treatment of depression. Ketamine is unique in the class of general anesthetics for a number of reasons. At the molecular level, the $\gamma$-aminobutyric acid $(\mathrm{GABA})_{\mathrm{A}}$ receptor is not the primary target for ketamine, unlike many drugs used for the induction and maintenance of general anesthesia. Rather, ketamine is thought to act by antagonizing glutamatergic Nmethyl-D-aspartate (NMDA) receptors (like the related anesthetics nitrous oxide and xenon) and/or hyperpolarization-activated cyclic-nucleotide gated (HCN) 1 channels (Yamamura et al., 1990; Chen et al., 2009; Zhou et al., 2013). At the neurochemical level, ketamine is unique because it increases cortical acetylcholine levels and appears to depend on noradrenergic signaling for its effects, in contrast to a number of GABAergic anesthetics (Kikuchi et al., 1997; Kushikata et al., 2011). At the systems neuroscience level, ketamine is also distinct because it does not metabolically activate the ventrolateral preoptic nucleus, a sleep-promoting nucleus in the hypothalamus that is activated by commonly-used anesthetics such as propofol and isoflurane; instead, it activates the wake-promoting locus coeruleus (Lu et al., 2008). Furthermore, in contrast to virtually all other anesthetic and sedative drugs, ketamine does not appear to metabolically depress the thalamus (Långsjö et al., 2005). Finally, at the neurophysiologic level, ketamine tends to increase the power of high-frequency electroencephalographic activity, whereas most anesthetics depress this bandwidth (Maksimow et al., 2006). Ketamine therefore fails to conform to virtually all mechanistic frameworks of anesthetic-induced unconsciousness.

Despite the many unique characteristics of ketamine, we recently demonstrated that anesthetic doses of ketamine selectively inhibit frontal-to-parietal directed connectivity, as measured by symbolic transfer entropy in electroencephalographic signals (Lee et al., 2013b). This finding was notable because selective inhibition of effective and directional connectivity from frontal to parietal regions has been demonstrated for two commonly-used anesthetics that act (at least in part) through GABA receptors: propofol [demonstrated with symbolic transfer entropy, evolution map approach, dynamic causal modeling, directed phase lag index (dPLI)] and sevoflurane (demonstrated with symbolic transfer entropy, evolution map approach) (Lee et al., 2009, 2013a; Ku et al., 2011; Boly et al., 2012). Given the proposed role of top-down reentrant processing and frontal-parietal 
networks in the conscious perception of environmental stimuli (Dehaene and Changeux, 2011; Demertzi et al., 2013), this finding suggests the exciting possibility of a common neurobiology underlying anesthetic-induced unconsciousness.

In the current study, we further characterized the effects of ketamine on the electroencephalogram, with a focus on alpha rhythms $(8-14 \mathrm{~Hz})$. Alpha oscillations have been an active area of research into anesthetic-induced unconsciousness because of a characteristic process referred to as anteriorization. In the resting/eyes-closed state in humans, the electroencephalographic power of alpha is dominant over the occipital cortex. However, at the point of propofol-induced unconsciousness, the power of alpha is dominant over the frontal cortex (Feshchenko et al., 2004; Purdon et al., 2013). Computational models that account for the observed posterior-to-anterior shift of alpha rhythms are based on propofol's agonism of $\mathrm{GABA}_{\mathrm{A}}$ receptors in the thalamus (Ching et al., 2010; Vijayan et al., 2013; Ching and Brown, 2014). The anteriorization of alpha at the point of propofolinduced unconsciousness also appears to relate to a characteristic cross-frequency coupling pattern between the amplitude of alpha and the phase of slow oscillations. During transitions into and out of propofol-induced unconsciousness, there is a troughmax relationship, i.e., the maximal alpha amplitude is coupled to the trough of the slow oscillation. During deeper levels of propofol-induced unconsciousness, however, this coupling shifts to a peak-max relationship (Purdon et al., 2013; Mukamel et al., 2014). These coupling patterns have not been investigated during unconsciousness induced by ketamine or other non-GABAergic anesthetics.

The propofol-induced shift to hypersynchronous alpha in the frontal cortex has been posited to impair flexible corticocortical communication (Supp et al., 2011), a process thought to be critical for consciousness. This hypothesis is supported by our recent work demonstrating that propofol-induced unconsciousness is characterized by a depression of anterior-to-posterior corticocortical connectivity (as measured by dPLI) that is found only in the alpha bandwidth (Lee et al., 2013a). dPLI is a measure of directional connectivity that appears to be closely related to alpha, since neural mass models of the human brain demonstrate an anterior-to-posterior flow of dPLI in the alpha bandwidth (Stam and van Straaten, 2012). This finding has been confirmed empirically by our previous study of dPLI in the resting state of conscious human volunteers (Lee et al., 2013a).

The potential dependence of anteriorized alpha rhythms on $\mathrm{GABA}_{\mathrm{A}}$ effects in the thalamus leads to the prediction that a non-GABAergic drug like ketamine would differ significantly from propofol and not increase the power of frontal alpha rhythms in association with the induction of unconsciousness. Similarly, we would not predict any characteristic cross-frequency coupling patterns around the time of unconsciousness, since these relationships appear to depend on increases in the frontal power of alpha. However, if the unconscious states induced by ketamine and propofol nonetheless share an underlying neurobiology, we would predict that the directional connectivity measure of dPLI would be inhibited by ketamine (as is observed with propofol), both in terms of the specificity of the alpha bandwidth and the anterior-to-posterior directionality. We tested these predictions regarding the effects of ketamine on alpha oscillations by re-analyzing electroencephalographic data acquired during consciousness and ketamine-induced unconsciousness in human surgical patients.

\section{METHODS \\ PARTICIPANTS}

We collected electroencephalographic data from 30 patients undergoing elective stomach, colorectal, thyroid or breast surgery (15 males; American Society Anesthesiologists Physical Status 1 or 2; 22-64 years old) at the Asan Medical Center (Seoul, South Korea). This study was approved by the Institutional Review Board of Asan Medical Center; written consent was provided by all participants after a careful discussion of risks and benefits. Patients were excluded from the study if they had a history of cardiovascular disease (including hypertension), brain surgery, drug/alcohol dependence, neurological or psychiatric disorder, or if they were currently using psychotropic medication. The data collected were previously analyzed and published (Lee et al., 2013b); the current study uses distinct analytic methods to test distinct hypotheses. For this secondary analysis, two participants were excluded due to insufficient artifact-free data in the baseline recording period; accordingly, data from 28 participants were analyzed at the University of Michigan Medical School (Ann Arbor, MI) for the current study.

\section{ANESTHETIC PROTOCOL}

Unconsciousness was induced using an infusion of ketamine $(2 \mathrm{mg} / \mathrm{kg}$ diluted in $10 \mathrm{~mL}$ of $0.9 \%$ normal saline) over a 2 min period (Baxter infusion pumpAS40A, Baxter Healthcare Corporation, Deerfield, IL). Consciousness was monitored by assessing the participant's response to an auditory command ("squeeze your right hand twice"), which was repeated every $10 \mathrm{~s}$. Prior to loss of consciousness, no sedatives or other medications were administered to the participant. Standard intraoperative monitors (non-invasive blood pressure measurement, electrocardiography, pulse oximetry and end-tidal carbon dioxide concentration and non-invasive) were used throughout the experiment; if systolic blood pressure increased to over $30 \%$ of baseline values, 5-10 mg of labetalol were administered.

\section{ELECTROENCEPHALOGRAM DATA ACOUISITION}

The electroencephalogram was acquired at 8 electrodes located at Fp1, Fp2, F3, F4, T3, T4, P3, and P4 (International 10-20 system); all channels were referenced to A2. Electrode impedances were reduced to below $5 \mathrm{~K} \Omega$ prior to data collection and electroencephalographic signals were collected used a Laxtha WEEG-32 amplifier (LXE3232-RF, Laxtha Inc., Daejeon, Korea) with a sampling frequency of $256 \mathrm{~Hz}$. Data recording began 5 min prior to induction; during this period, participants were instructed to rest with their eyes closed. Data collection continued through anesthetic induction and was terminated five minutes following loss of consciousness.

\section{ELECTROENCEPHALOGRAM ANALYSIS}

Preprocessing: Electroencephalogram data were bandpass filtered between 0.1 and $50 \mathrm{~Hz}$. The filtered data were visually 
inspected and non-physiological artifacts were removed. For the spectral and cross-frequency coupling analysis, two 5-min epochs of data were extracted from every participant: (1) Baseline rest, prior to ketamine induction, and (2) Induction and unconsciousness, beginning at the start of ketamine infusion. This second epoch was chosen specifically so that the transition from consciousness to unconsciousness could be studied, as some spectrographic and phase-amplitude coupling patterns appear during this period (Purdon et al., 2013). On average, participants lost consciousness $89 \pm 14 \mathrm{~s}$ after the start of ketamine infusion; thus, this epoch contains electroencephalographic data from both conscious and unconscious states. However, coherence, phase lag index (PLI) and dPLI measures were calculated only with the data after ketamine-induced consciousness. Spectral Analysis: Spectrograms were computed in Chronux (www. chronux.org), using the multitaper method, with window lengths of $T=2 \mathrm{~s}$, step size of $0.1 \mathrm{~s}$, time-bandwidth product $N W=$ 2 , number of tapers $K=3$. Group spectrograms were calculated by aggregating data from frontal channels F3 and F4 across all participants. Phase-Amplitude Coupling Analysis: Phaseamplitude coupling was conducted using the Phase-Amplitude Coupling Toolbox (PACT) in EEGlab (Delorme and Makeig, 2004; Miyakoshi et al., 2013). Finite impulse response filters were used to extract low-frequency $(0.1-1 \mathrm{~Hz})$ and alpha (8$14 \mathrm{~Hz}$ ) oscillations from channels F3 and F4 for each participant. Instantaneous phase and amplitude were extracted using a Hilbert transform. Phase-amplitude modulograms were calculated by assigning each temporal sample to one of $N=18$ equally spaced phase bins based on the instantaneous value of the lowfrequency phase and then averaging the corresponding instantaneous amplitude of alpha within a 1 min epoch. Additionally, Canolty's phase-amplitude coupling modulation index was calculated for each minute of data and averaged across all participants (Canolty et al., 2006). Functional Connectivity Analysis with Coherence: The magnitude squared coherence estimate was calculated between each set of channel combinations using Welch's averaged modified periodogram method (Welch, 1967). Functional Connectivity Analysis with Phase Lag Index (PLI): To mitigate the effects of choice of reference and volume conduction, we calculated functional connectivity using the metric PLI (Stam et al., 2007). We used a Hilbert transform to extract the instantaneous phase of the electroencephalogram from each channel and calculated the phase difference $\Delta \phi_{t}$ between channels, where $\Delta \phi_{t}=\phi_{i, t}-\phi_{j, t}, t=1,2, \ldots, \mathrm{n} \mathrm{n}$ is the number of samples within one epoch, and $i$ and $j$ were set to include all possible channel combinations. PLI was then calculated using Equation (1):

$$
\operatorname{PLI}_{i j}=\left|\left\langle\operatorname{sign}\left(\Delta \phi_{t}\right)\right\rangle\right|, \quad 0 \leq \mathrm{PLI}_{i j} \leq 1 .
$$

Here, the sign() function yields: 1 if $\Delta \phi_{t}>0$; 0 if $\Delta \phi_{t}=0$; and -1 if $\Delta \phi_{t}<0$. If the instantaneous phase of one signal is consistently ahead of the other signal, the phases are considered locked, and $\mathrm{PLI} \approx 1$. However, if the signals randomly alternate between a phase lead and a phase lag relationship, there is no phase locking and PLI $\approx 0$. Directed Connectivity Analysis with Directed Phase Lag Index (dPLI): To determine the direction of the phase-lead/phase-lag relationship between channels, we calculated dPLI between signals $i$ and $j$ using Equation (2) (Stam and van Straaten, 2012):

$$
\mathrm{dPLI}_{i j}=\left\langle H\left(\Delta \pi_{t}\right)\right\rangle
$$

Here, $H(\mathrm{x})$ represents the Heaviside step function, where $H(\mathrm{x})=$ lif $x>0, H(\mathrm{x})=0.5$ if $x=0$, and $H(\mathrm{x})=0$ otherwise. If, on average, signal $i$ leads signal $j, 0.5<\operatorname{dPLI}_{i j}=1$; if signal $j$ leads signal $i, 0=\mathrm{dPLI}_{i j}<0.5$; and if there is no phase-lead/phaselag relationship between signals, $\mathrm{dPLI}=0.5$. Surrogate Data Analysis: To quantify the effects of spurious phase relationships and power spectrum changes on functional and directed connectivity metrics, we generated surrogate data sets as follows. We calculated the instantaneous phase of each combination of channel pairs $i$ and $j$ for each epoch (baseline; ketamine-induced unconsciousness) using a Hilbert transform. The phase time series of channel $i$ was maintained, whereas in channel $j$, the phase time series from 0 to $n / 2$ was interchanged with the phase time series from $n / 2$ to $n$, where $n$ is the number of samples within one epoch. In this manner, existing phase relationships were eliminated while maintaining the spectral properties of each condition. PLI and dPLI were calculated for all channel combinations in the surrogate dataset. Brain Network Visualization: The brain networks were visualized with the BrainNet Viewer (Xia et al., 2013) (http://www.nitrc.org/projects/bnv/). Montreal Neurological Institute (MNI) coordinates of each scalp electrode were used to generate nodes that were projected onto an axial view of the brain. Coherence, PLI, and dPLI measures were indicated by varying the color and size of edges between these nodes.

\section{STATISTICAL ANALYSIS}

Phase Amplitude Coupling: To test the null hypothesis that the phase and amplitude of the frontal electroencephalogram are decoupled, we employed the surrogate data method proposed in Canolty et al. (2006). Data were divided into 1-min epochs; within each epoch, a surrogate time series was generated by shifting the amplitude by a random time $\Delta \mathrm{t}(0 \leq \Delta \mathrm{t} \leq 60 \mathrm{~s})$ while keeping the phase series fixed. The modulation index was calculated for the surrogate series. This procedure was repeated 2000 times to produce distributions of modulation index values in which the null hypothesis was true. The modulation index for the experimental data was considered significant if it exceeded $95 \%$ of the surrogate values $(p<0.05)$. Coherence, PLI, and dPLI: Coherence, PLI, and dPLI values were compared between (1) baseline and unconscious epochs, (2) baseline and surrogate baseline epochs, and (3) unconscious and surrogate unconscious epochs using a student's $t$-test. Differences were considered significant at $\alpha<0.05$.

\section{RESULTS}

\section{KETAMINE-INDUCED UNCONSCIOUSNESS IS NOT CHARACTERIZED BY INCREASES IN FRONTAL ALPHA POWER OR BY PHASE-AMPLITUDE COUPLING PATTERNS}

The group-level spectrogram of the electroencephalogram recorded from frontal channels demonstrates a decrease in alpha power upon ketamine-induced unconsciousness (Figure 1). This 


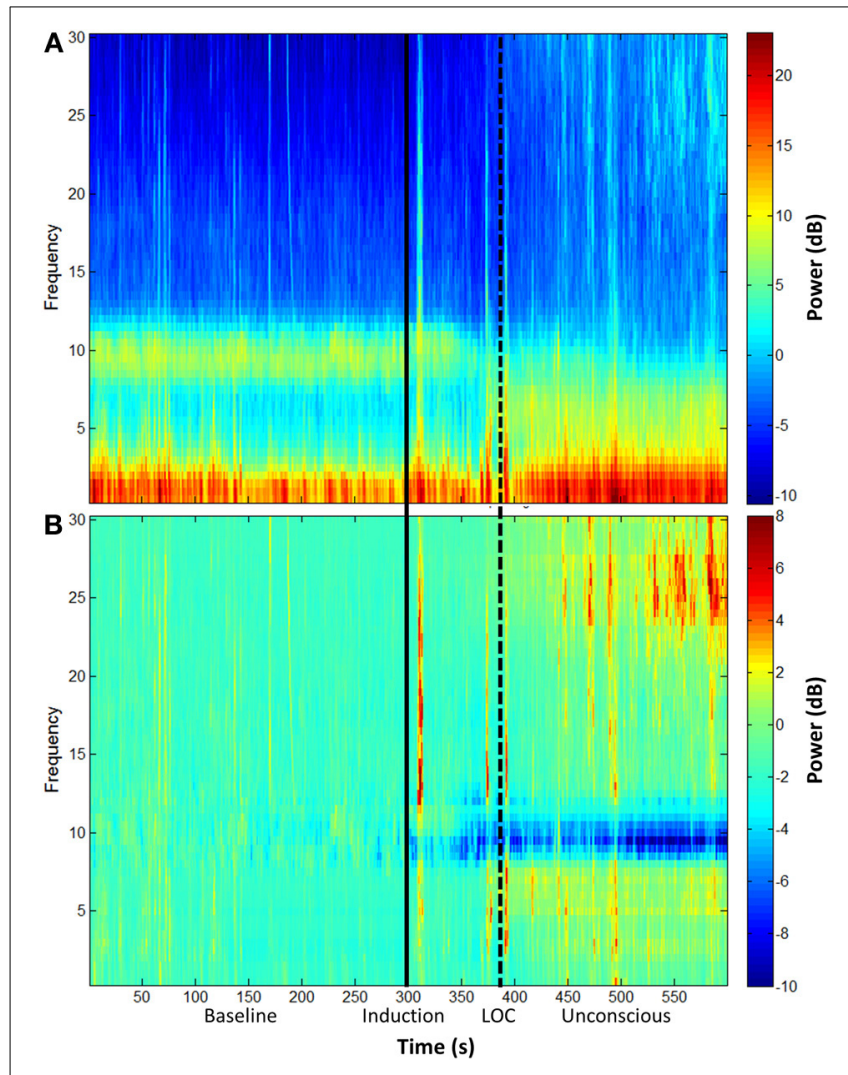

FIGURE 1 | Group spectral analysis. (A) Spectrogram and (B) baseline-normalized spectrogram of frontal channels (F3 and F4) demonstrate a decrease in alpha power during and after ketamine-induced unconsciousness. LOC, loss of consciousness.

is the opposite pattern to that observed in propofol-induced unconsciousness, which is associated with an increase in alpha power (Purdon et al., 2013).

Cross-frequency coupling patterns were calculated between slow oscillation phase and alpha amplitude in the frontal channels. Group modulograms and the group-averaged modulation index demonstrate that a significant trough-max coupling pattern existed for $40 \%$ of the baseline resting state, but no significant coupling was observed upon induction or after loss of consciousness (Figure 2). These patterns are in contrast to those observed with propofol, where loss of consciousness is associated with a trough-max coupling pattern (Mukamel et al., 2014). It is worthwhile noting that when the test for phase-amplitude coupling significance (Canolty et al., 2006) is applied to datasets with minimal to no cross-frequency coupling patterns, epochs with even moderate coupling patterns will exceed the modulation index associated with significance. This may explain the significant phase-amplitude coupling observed during the resting state of our study, which has not been observed in previous studies.

\section{KETAMINE-INDUCED UNCONSCIOUSNESS IS ASSOCIATED WITH DECREASES IN PLI, BUT NOT IN COHERENCE}

We examined two measures of functional connectivity in the alpha bandwidth between frontal and parietal channels:

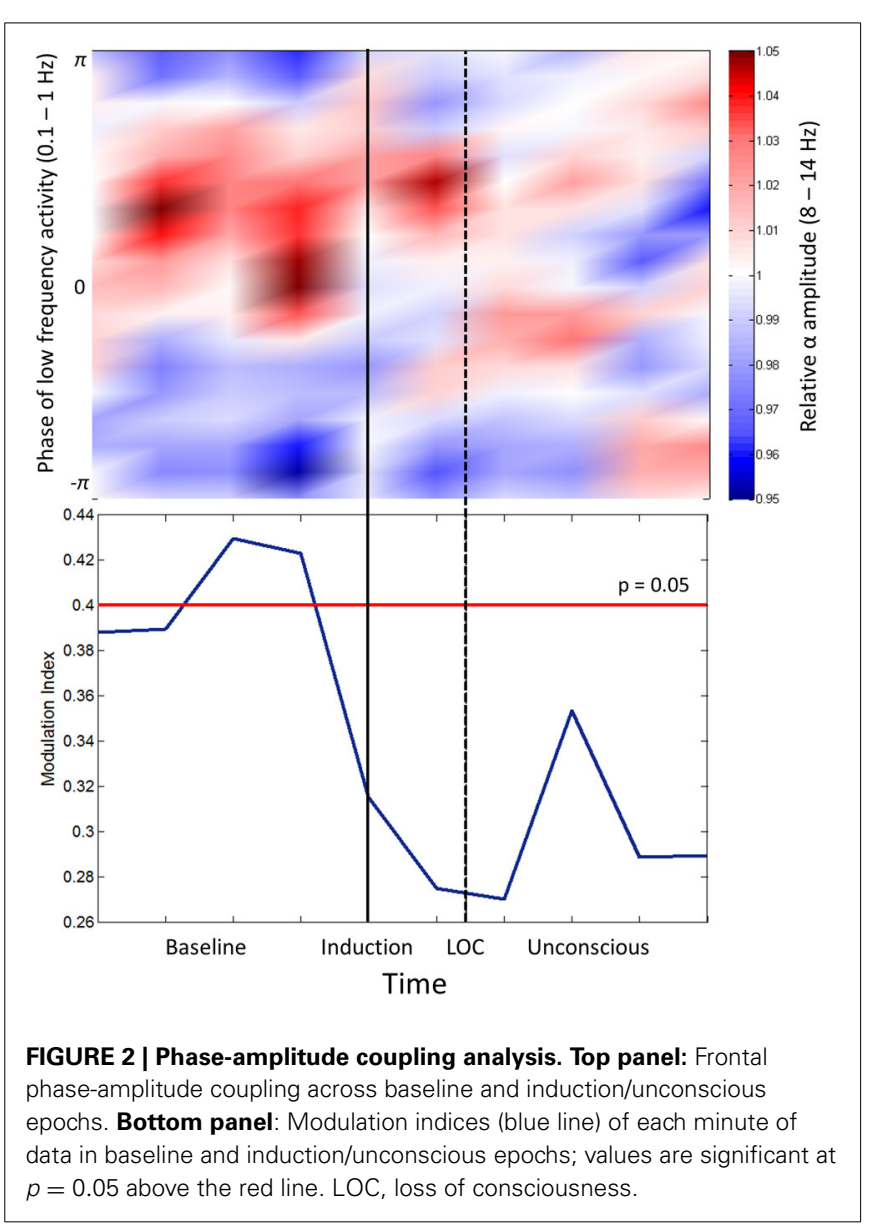

coherence and PLI. We observed no significant change in frontoparietal coherence $(p=0.29)$ between baseline resting and ketamine-induced unconsciousness (Figure 3).

In contrast, PLI decreased upon ketamine-induced unconsciousness. The largest decrease in PLI was observed in the alpha bandwidth, where PLI values during unconsciousness were significantly lower than PLI values calculated during baseline consciousness $(p<0.001)$ (Figures 4A,B). PLI values measured from surrogate data in both epochs were $\sim 0$, indicating that the values calculated from experimental data are not the result of spurious phase relationships. While there was an overall decrease in PLI across all channel combinations, the largest decrease occurred across frontoparietal channel pairs (Figure 4C).

\section{KETAMINE-INDUCED UNCONSCIOUSNESS IS ASSOCIATED WITH DECREASED FRONTOPARIETAL dPLI}

We examined differences in dPLI between baseline resting and unconscious states. The greatest differences in dPLI occurred in the alpha bandwidth (Figure 5A) and a significant decrease in APLI from frontal to parietal brain regions $(p<0.001)$ was observed between states (Figure 5B). There was no significant difference between dPLI values of the surrogate data generated from either state, indicating that the observed changes in dPLI for the experimental data cannot be attributed to changes in spectral power between conditions. Baseline dPLI indicated 

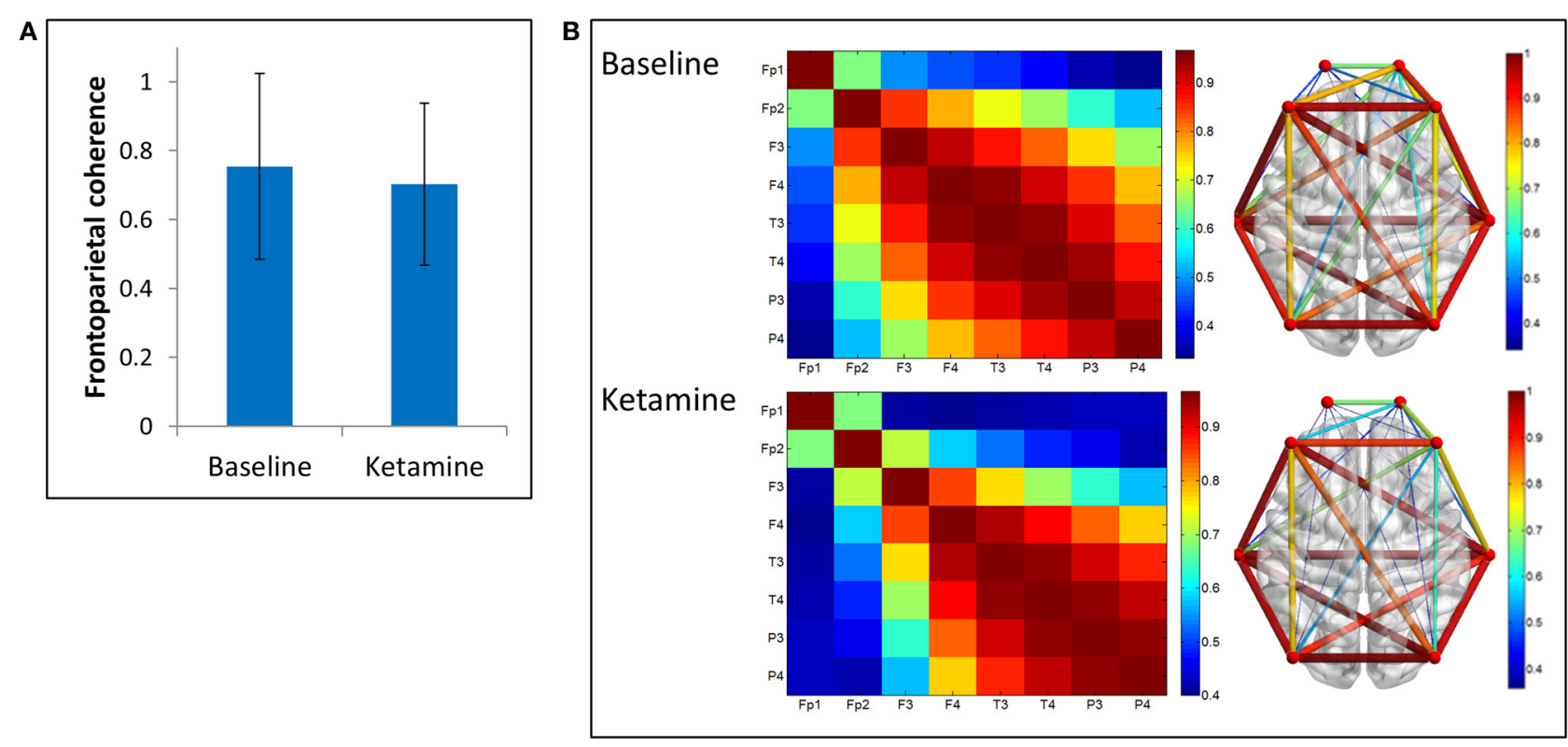

FIGURE 3 | Coherence analysis. (A) Average frontoparietal coherence across 28 participants in baseline resting and ketamine-induced unconscious states (error bars indicate standard deviation). No significant differences are observed in coherence between conditions. (B) Average coherence across all channel combinations in baseline rest and ketamine-induced unconscious states, represented on a channel grid (left) and on an axial view of the brain (right). On the brain network, both color and size of the edges represent strength of coherence. that the dominant direction of connectivity is from frontal to parietal regions when participants are conscious; upon ketamineinduced unconsciousness there is a balance of frontoparietal and parietofrontal directional connectivity (Figure 5C).

\section{DISCUSSION}

This study of ketamine and alpha oscillations revealed both drugrelated and state-related effects in association with the induction of unconsciousness. Unlike propofol, ketamine does not increase frontal alpha power or induce characteristic cross-frequency coupling patterns between the power of alpha and the phase of slowwave oscillations. Functional connectivity in the alpha bandwidth was preserved between states when measured by coherence, but decreased upon unconsciousness when measured by PLI. The discrepancy between these two functional connectivity metrics indicates that while the correlation between electroencephalogram signals in frontal and parietal regions remains unchanged in aggregate, their specific phase-relationship is disrupted upon loss of consciousness. Directional connectivity in the alpha bandwidth (as measured by dPLI) was inhibited across the frontoparietal network by ketamine, a finding consistent with our past study of propofol-induced unconsciousness (Lee et al., 2013a). The identification of shared neural features between the unconscious states induced by GABAergic and non-GABAergic anesthetics has been a longstanding problem in the study of both anesthetic mechanisms and anesthetic monitoring. The current findings support the hypothesis that anesthetic-induced unconsciousness has a common neurobiology related to disrupted functional relationships across cortical or thalamocortical networks.

Activity and connectivity of lateral frontal and posterior parietal cortices have been hypothesized to be critical for consciousness of environmental stimuli (Boly et al., 2007; Demertzi et al., 2013). As such, the finding that a variety of general anesthetic drugs suppresses both activity and connectivity in these regions appears relevant to the proximate cause of anesthetic-induced unconsciousness. Prior preclinical studies of anterior-posterior connectivity in rat brain identified a selective inhibition of frontal-to-posterior transfer entropy in the gamma bandwidth in association with isoflurane-induced unconsciousness (Imas et al., 2005). Our laboratory first demonstrated anesthetic inhibition of frontal-to-parietal connectivity in human volunteers (Lee et al., 2009) and surgical patients (Ku et al., 2011); inhibition of functional, directional, and effective connectivity in frontal-parietal networks in association with propofol-induced unconsciousness has been identified by studies from multiple research groups using multiple analytic methods (Boveroux et al., 2010; Schrouff et al., 2011; Boly et al., 2012; Jordan et al., 2013). Of note, the recent study of Jordan et al used combined electroencephalography and functional magnetic resonance imaging with no a priori assumptions regarding connectivity and found that the selective loss of frontal-to-parietal connectivity (as measured by symbolic transfer entropy) was the best discriminator between consciousness and propofol-induced unconsciousness (Jordan et al., 2013). However, most studies of directional/effective connectivity in frontal-parietal networks have focused on propofol, a prototypical $\mathrm{GABA}_{\mathrm{A}}$ agonist. Recently, our laboratory demonstrated a similar and selective inhibition of frontal-to-parietal connectivity during unconsciousness induced by the diverse anesthetics ketamine, propofol, and sevoflurane (Lee et al., 2013b). This study was the first to identify a common network-level disruption induced by both GABAergic and non-GABAergic anesthetics. The 


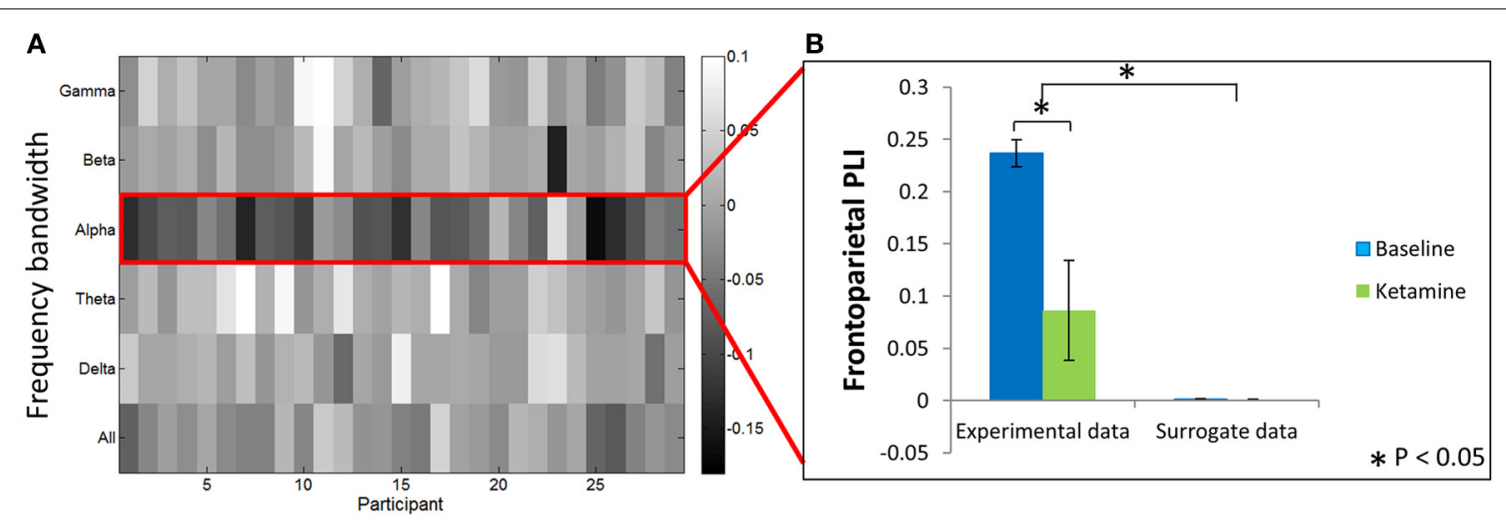

C

FIGURE 4 | Phase lag index analysis. (A) Difference in PLI between baseline and ketamine-induced unconscious states represented for all bandwidths, across all participants. The largest decreases in PLI are observed in the alpha bandwidth. (B) Average frontoparietal PLI values for experimental data and surrogate data (error bars indicate standard deviation). Frontoparietal PLI significantly decreases (as indicated by ${ }^{*}$ ) between states in the experimental data; no phase-locking is observed in the surrogate dataset. (C) Average difference in PLI between baseline and unconscious states across all channel combinations, represented on a channel grid (left), and on the axial view of the brain (right). Color and size of edges in the brain network represent the difference in PLI between baseline and unconscious states. Large decreases in PLI are observed between frontoparietal channel combinations. current study builds on this work by using alternative connectivity measures to support the hypothesis that anesthetic-induced unconsciousness is characterized by interrupted directed connectivity from frontal to parietal regions. Impaired connectivity is of relevance to the mechanism of anesthetic-induced unconsciousness because it reduces the likelihood of achieving the neural information synthesis thought to be necessary for conscious perception (Tononi, 2011). Furthermore, by comparing ketamine's effects on the alpha bandwidth to past studies of propofol, we are now able to distinguish between drug-related and state-related effects on the electroencephalogram. Despite the divergent spectral effects on alpha oscillations induced by ketamine in this study and those reported in past studies of propofol, disruption of functional (PLI) and directional (dPLI) connectivity in the alpha bandwidth appears common to the state of unconsciousness induced by both drugs. It should be noted that changes in dPLI were reversed upon recovery from propofol; given the current study design we were not able to assess recovery of directional connectivity for ketamine.
This study has numerous limitations. First, we reanalyzed electroencephalographic data from a prior study of ketamine-induced unconsciousness (Lee et al., 2013b). It might be argued that the current findings could have been predicted from our past methodology using symbolic transfer entropy. However, this is not necessarily the case, since analysis of the same electroencephalographic dataset (Murphy et al., 2011) with dynamic causal modeling (Boly et al., 2012) and Granger causality (Barrett et al., 2012) yielded discrepant results regarding directionality across frontal and parietal cortices. Second, we used low-resolution electroencephalography given the clinical nature of the original study; thus, source-localized signals could not be analyzed. Third, there was no recording from occipital electrodes, which precluded topographic analysis that would allow comparison of alpha power in posterior and anterior structures. Fourth, it could be argued that the state of ketamine-induced unconsciousness is substantially distinct from the state of propofolinduced unconsciousness, which renders any comparisons questionable. Although it is true that ketamine-based anesthetics can 

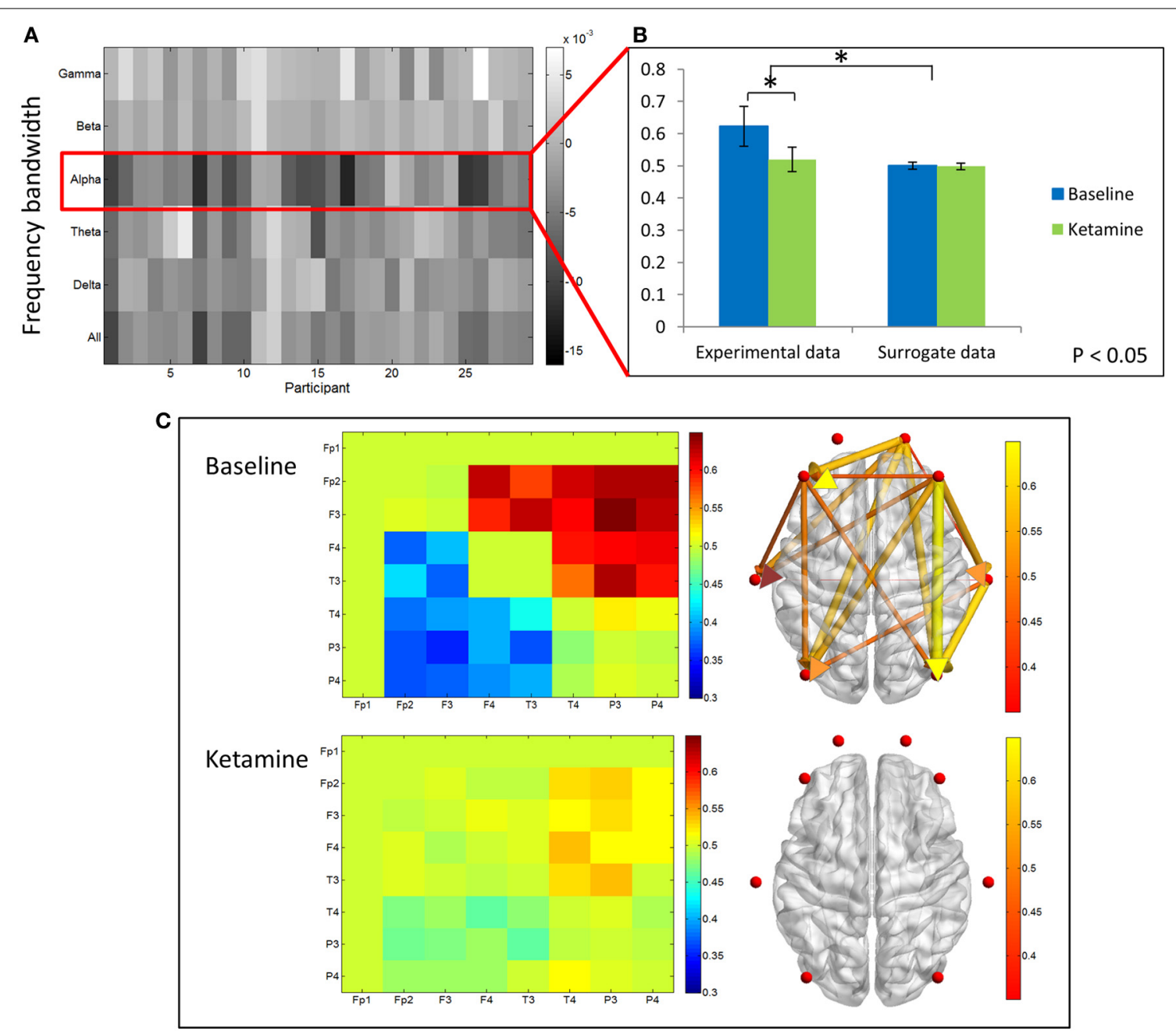

FIGURE 5 | Directed phase lag index analysis. (A) Difference in frontoparietal dPLI between baseline rest and ketamine-induced unconscious states, represented across six frequency bandwidths and all participants. The largest decreases in dPLI occur in the alpha bandwidth. Note that a dPLI value of 0.5 indicates neither phase lag nor phase lead relationship. (B) Average frontoparietal dPLI values for each state in experimental and surrogate data (error bars indicate standard deviation). Frontoparietal dPLI significantly decreases during ketamine-induced unconsciousness (as indicated by *). Surrogate data demonstrate no phase-lead/phase-lag relationship in either state. (C) Average dPLI values across all channel combinations in baseline and unconscious states. Only dPLI values greater than 0.55 are represented on the axial brain network. In this network, color, and size of the edges indicate the dPLI value between two nodes; arrows indicate the direction of phase lead and lag. be associated with conscious states such as dreaming or hallucinations (Grace, 2003), the same could be argued-albeit to a lesser degree-for propofol (Leslie et al., 2007). Furthermore, from the functional perspective, both ketamine and propofol can be used to induce unconsciousness in clinical settings; this functional similarity motivates the search for common mechanisms. Fifth, it one could argue that the reduction in PLI and DPLI we observed in the alpha bandwidth during induction/unconsciousness can be attributed to the dramatic decrease in power upon loss of consciousness. However, we have demonstrated that PLI and dPLI values calculated for surrogate datasets that have the same spectral characteristics are not significantly different. Furthermore, the fact that coherence values are not significantly different between states demonstrates that the change in PLI and dPLI values cannot be attributed to reduced power in the alpha bandwidth. Finally, we used loss of responsiveness as a surrogate for loss of consciousness; it is well known that consciousness and responsiveness are dissociable. However, this is a limitation common to all studies of anesthetic-induced unconsciousness. Although, as noted, loss of responsiveness induced by ketamine may still be associated with conscious states such as dreams, it is the connection to the environment that is likely of primary clinical relevance (Sanders et al., 2012).

Despite these limitations, this analysis of ketamine's effects on spectral changes, cross-frequency coupling, and connectivity in the alpha bandwidth provides insight into the neurobiology of ketamine-induced unconsciousness. In comparison with past studies of propofol, the distinct effects of ketamine suggest that the ability to induce anteriorized alpha and cross-frequency coupling varies across anesthetic drugs. By contrast, disrupted 
directional connectivity in the frontal-parietal network may be a common state-related feature-and, potentially, a common mechanism—of anesthetic-induced unconsciousness.

\section{FUNDING}

Supported by departmental and institutional funds.

\section{REFERENCES}

Barrett, A. B., Murphy, M., Bruno, M.-A., Noirhomme, Q., Boly, M., Laureys, S., et al. (2012). Granger causality analysis of steady-state electroencephalographic signals during propofol-induced anaesthesia. PLoS ONE 7:e29072. doi: 10.1371/journal.pone.0029072

Boly, M., Balteau, E., Schnakers, C., Degueldre, C., Moonen, G., Luxen, A., et al. (2007). Baseline brain activity fluctuations predict somatosensory perception in humans. Proc. Natl. Acad. Sci. U.S.A. 104, 12187-12192. doi: 10.1073/pnas.0611404104

Boly, M., Moran, R., Murphy, M., Boveroux, P., Bruno, M.-A., Noirhomme, Q., et al. (2012). Connectivity changes underlying spectral EEG changes during propofol-induced loss of consciousness. J. Neurosci. 32, 7082-7090. doi: 10.1523/JNEUROSCI.3769-11.2012

Boveroux, P., Vanhaudenhuyse, A., Bruno, M.-A., Noirhomme, Q., Lauwick, S., Luxen, A., et al. (2010). Breakdown of within- and between-network resting state functional magnetic resonance imaging connectivity during propofol-induced loss of consciousness. Anesthesiology 113, 1038-1053. doi: 10.1097/ALN.0b013e3181f697f5

Canolty, R. T., Edwards, E., Dalal, S. S., Soltani, M., Nagarajan, S. S., Kirsch, H. E., et al. (2006). High gamma power is phase-locked to theta oscillations in human neocortex. Science 313, 1626-1628. doi: 10.1126/science.1128115

Chen, X., Shu, S., and Bayliss, D. A. (2009). HCN1 channel subunits are a molecular substrate for hypnotic actions of ketamine. J. Neurosci. 29, 600-609. doi: 10.1523/JNEUROSCI.3481-08.2009

Ching, S., and Brown, E. N. (2014). Modeling the dynamical effects of anesthesia on brain circuits. Curr. Opin. Neurobiol. 25, 116-122. doi: 10.1016/j.conb.2013.12.011

Ching, S., Cimenser, A., Purdon, P. L., Brown, E. N., and Kopell, N. J. (2010). Thalamocortical model for a propofol-induced $\alpha$-rhythm associated with loss of consciousness. Proc. Natl. Acad. Sci. U.S.A. 107, 22665-22670. doi: $10.1073 /$ pnas. 1017069108

Corssen, G., and Domino, E. F. (1966). Dissociative anesthesia: further pharmacologic studies and first clinical experience with the phencyclidine derivative CI-581. Anesth. Analg. 45, 29-40.

Dehaene, S., and Changeux, J.-P. (2011). Experimental and theoretical approaches to conscious processing. Neuron 70, 200-227. doi: 10.1016/j.neuron.2011.03.018

Delorme, A., and Makeig, S. (2004). EEGLAB: an open source toolbox for analysis of single-trial EEG dynamics including independent component analysis. J. Neurosci. Methods 134, 9-21. doi: 10.1016/j.jneumeth.2003.10.009

Demertzi, A., Soddu, A., and Laureys, S. (2013). Consciousness supporting networks. Curr. Opin. Neurobiol. 23, 239-211. doi: 10.1016/j.conb.2012. 12.003

Feshchenko, V. A., Veselis, R. A., and Reinsel, R. A. (2004). Propofol-induced alpha rhythm. Neuropsychobiology 50, 257-266. doi: 10.1159/000079981

Grace, R. F. (2003). The effect of variable-dose diazepam on dreaming and emergence phenomena in 400 cases of ketamine-fentanyl anaesthesia. Anaesthesia 58, 904-910. doi: 10.1046/j.1365-2044.2003.03341.x

Imas, O. A., Ropella, K. M., Ward, B. D., Wood, J. D., and Hudetz, A. G. (2005). Volatile anesthetics disrupt frontal-posterior recurrent information transfer at gamma frequencies in rat. Neurosci. Lett. 387, 145-150. doi: 10.1016/j.neulet.2005.06.018

Jordan, D., Ilg, R., Riedl, V., Schorer, A., Grimberg, S., Neufang, S., et al. (2013). Simultaneous electroencephalographic and functional magnetic resonance imaging indicate impaired cortical top-down processing in association with anesthetic-induced unconsciousness. Anesthesiology 119, 1031-1042. doi: 10.1097/ALN.0b013e3182a7ca92

Kikuchi, T., Wang, Y., Shinbori, H., Sato, K., and Okumura, F. (1997). Effects of ketamine and pentobarbitone on acetylcholine release from the rat frontal cortex in vivo. Br. J. Anaesth. 79, 128-130.
Ku, S.-W., Lee, U., Noh, G.-J., Jun, I.-G., and Mashour, G. A. (2011). Preferential inhibition of frontal-to-parietal feedback connectivity is a neurophysiologic correlate of general anesthesia in surgical patients. PLOS ONE 6:e25155. doi: 10.1371/journal.pone.0025155

Kushikata, T., Yoshida, H., Kudo, M., Kudo, T., Kudo, T., and Hirota, K. (2011). Role of coerulean noradrenergic neurones in general anaesthesia in rats. Br. J. Anaesth. 107, 924-929. doi: 10.1093/bja/aer303

Långsjö, J. W., Maksimow, A., Salmi, E., Kaisti, K., Aalto, S., Oikonen, V., et al. (2005). S-ketamine anesthesia increases cerebral blood flow in excess of the metabolic needs in humans. Anesthesiology 103, 258-268. doi: 10.1097/00000542-200508000-00008

Lee, H., Mashour, G. A., Noh, G.-J., Kim, S., and Lee, U. (2013a). Reconfiguration of network hub structure after propofol-induced unconsciousness. Anesthesiology 119, 1347-1359. doi: 10.1097/ALN.0b013e3182a8ec8c

Lee, U., Kim, S., Noh, G.-J., Choi, B.-M., Hwang, E., and Mashour, G. A. (2009). The directionality and functional organization of frontoparietal connectivity during consciousness and anesthesia in humans. Conscious. Cogn. 18, 1069-1078. doi: 10.1016/j.concog.2009.04.004

Lee, U., Ku, S., Noh, G., Baek, S., Choi, B., and Mashour, G. A. (2013b). Disruption of frontal-parietal communication by ketamine, propofol, and sevoflurane. Anesthesiology 118, 1264-1275. doi: 10.1097/ALN.0b013e3182 $9103 \mathrm{f} 5$

Leslie, K., Skrzypek, H., Paech, M., Kurowski, I., and Whybrow, T. (2007). Dreaming during anesthesia and anesthetic depth in elective surgery patients: a prospective cohort study. Anesthesiology 106, 33-42. doi: 10.1097/00000542200701000-00010

Lu, J., Nelson, L. E., Franks, N., Maze, M., Chamberlin, N. L., and Saper, C. B. (2008). Role of endogenous sleep-wake and analgesic systems in anesthesia. J. Comp. Neurol. 508, 648-662. doi: 10.1002/cne.21685

Maksimow, A., Särkelä, M., Långsjö, J. W., Salmi, E., Kaisti, K. K., Yli-Hankala, A., et al. (2006). Increase in high frequency EEG activity explains the poor performance of EEG spectral entropy monitor during S-ketamine anesthesia. Clin. Neurophysiol. 117, 1660-1668. doi: 10.1016/j.clinph.2006. 05.011

Miyakoshi, M., Delorme, A., Mullen, T., Kojima, K., Makeig, S., and Asano, E. (2013). Automated detection of cross-frequency coupling in the electrocorticogram for clinical inspection. Conf. Proc. IEEE Eng. Med. Biol. Soc. 2013, 3282-3285. doi: 10.1109/EMBC.2013.6610242

Mukamel, E. A., Pirondini, E., Babadi, B., Wong, K. F. K., Pierce, E. T., Harrell, P. G., et al. (2014). A transition in brain state during propofol-induced unconsciousness. J. Neurosci. 34, 839-845. doi: 10.1523/JNEUROSCI.581312.2014

Murphy, M., Bruno, M.-A., Riedner, B. A., Boveroux, P., Noirhomme, Q., Landsness, E. C., et al. (2011). Propofol anesthesia and sleep: a high-density EEG study. Sleep 34, 283-291.

Purdon, P. L., Pierce, E. T., Mukamel, E. A., Prerau, M. J., Walsh, J. L., Wong, K. F. K., et al. (2013). Electroencephalogram signatures of loss and recovery of consciousness from propofol. Proc. Natl. Acad. Sci. U.S.A. 110, E1142-E1151. doi: $10.1073 /$ pnas. 1221180110

Sanders, R. D., Tononi, G., Laureys, S., and Sleigh, J. W. (2012). Unresponsiveness $\neq$ unconsciousness. Anesthesiology 116, 946-959. doi: 10.1097/ALN.0b013e3182 49d0a7

Schrouff, J., Perlbarg, V., Boly, M., Marrelec, G., Boveroux, P., Vanhaudenhuyse, A., et al. (2011). Brain functional integration decreases during propofol-induced loss of consciousness. Neuroimage 57, 198-205. doi: 10.1016/j.neuroimage.2011.04.020

Stam, C. J., Nolte, G., and Daffertshofer, A. (2007). Phase lag index: assessment of functional connectivity from multi channel EEG and MEG with diminished bias from common sources. Hum. Brain Mapp. 28, 1178-1193. doi: 10.1002/hbm.20346

Stam, C. J., and van Straaten, E. C. W. (2012). Go with the flow: use of a directed phase lag index (dPLI) to characterize patterns of phase relations in a large-scale model of brain dynamics. Neuroimage 62, 1415-1428. doi: 10.1016/j.neuroimage.2012.05.050

Supp, G. G., Siegel, M., Hipp, J. F., and Engel, A. K. (2011). Cortical hypersynchrony predicts breakdown of sensory processing during loss of consciousness. Curr. Biol. 21, 1988-1993. doi: 10.1016/j.cub.2011.10.017

Tononi, G. (2011). The integrated information theory of consciousness: an updated account. Arch. Ital. Biol. 150, 56-90. doi: 10.4449/aib.v149i5.1388 
Vijayan, S., Ching, S., Purdon, P. L., Brown, E. N., and Kopell, N. J. (2013). Thalamocortical mechanisms for the anteriorization of alpha rhythms during propofol-induced unconsciousness. J. Neurosci. 33, 11070-11075. doi: 10.1523/JNEUROSCI.5670-12.2013

Welch, P. D. (1967). The use of fast Fourier transform for the estimation of power spectra: a method based on time averaging over short, modified periodograms. IEEE Trans. Audio Electroacoustics 15, 70-73. doi: 10.1109/TAU.1967. 1161901

Xia, M., Wang, J., and He, Y. (2013). BrainNet Viewer: a network visualization tool for human brain connectomics. PLoS ONE 8:e68910. doi: 10.1371/journal.pone.0068910

Yamamura, T., Harada, K., Okamura, A., and Kemmotsu, O. (1990). Is the site of action of ketamine anesthesia the N-methyl-D-aspartate receptor? Anesthesiology 72, 704-710.

Zhou, C., Douglas, J. E., Kumar, N. N., Shu, S., Bayliss, D. A., and Chen, X. (2013). Forebrain HCN1 channels contribute to hypnotic actions of ketamine. Anesthesiology 118, 785-795. doi: 10.1097/ALN.0b013e318 $287 \mathrm{~b} 7 \mathrm{c} 8$
Conflict of Interest Statement: Dr. George A. Mashour and Dr. UnCheol Lee have a patent pending (through the University of Michigan, Ann Arbor) on the measurement of directional connectivity for monitoring consciousness. The authors declare that the research was conducted in the absence of any commercial or financial relationships that could be construed as a potential conflict of interest.

Received: 22 April 2014; accepted: 27 May 2014; published online: 01 July 2014.

Citation: Blain-Moraes S, Lee U, Ku S, Noh G and Mashour GA (2014) Electroencephalographic effects of ketamine on power, cross-frequency coupling, and connectivity in the alpha bandwidth. Front. Syst. Neurosci. 8:114. doi: 10.3389/fnsys. 2014.00114

This article was submitted to the journal Frontiers in Systems Neuroscience.

Copyright (C) 2014 Blain-Moraes, Lee, Ku, Noh and Mashour. This is an openaccess article distributed under the terms of the Creative Commons Attribution License (CC BY). The use, distribution or reproduction in other forums is permitted, provided the original author(s) or licensor are credited and that the original publication in this journal is cited, in accordance with accepted academic practice. No use, distribution or reproduction is permitted which does not comply with these terms. 